\title{
Neurotransmitter Chemistry of Lissencephalic Cortex Induced in Ferrets by Fetal Treatment with Methylazoxymethanol Acetate
}

\author{
M. V. JOHNSTON, R. HADDAD, A. CARMAN-YOUNG and J. T. COYLE \\ Department of Neurology, University of Michigan School of Medicine, Ann Arbor, MI 48109, (R.H.) \\ Neuroteratology Laboratory, New York State Institute for Basic Research in Mental Retardation, \\ Staten Island, NY 10314 and (A.C.-Y. and J.T.C.) Departments of Neuroscience, Pharmacology \\ and Experimental Therapeutics and Psychiatry and the Behavioral Sciences. \\ Johns Hopkins University, School of Medicine, \\ Baltimore, MD 21205 (U.S.A.)
}

(Accepted December 3rd, 1981)

Key words: ferret - lissencephalic cortex - methylazoxymethanol acetate

\begin{abstract}
Treatment of pregnant ferrets with $15 \mathrm{mg} / \mathrm{kg}$ of methylazoxymethanol acetate (MAM) at 33 days of fetal gestation results in off spring with cortical hypoplasia and lissencephally. Neurochemical analysis of 5 areas of cortex from 8-week-old offspring of MAMor vehicle-treated jills indicated an overall enrichment in markers for catecholaminergic (tyrosine hydroxylase, norepinephrine) and cholinergic (choline acetyltransferase, acetylcholine) terminals but minimal change in the concentration of GABAergic markers (glutamate decarboxylase, $\gamma$-aminobutyric acid); however, there did not appear to be a direct, inverse relationship between the concentration of catecholaminergic and cholinergic markers and the degree of hypoplasia in cortical subareas unlike what has been found previously in the rat.
\end{abstract}

\section{INTRODUCTION}

Methylazoxymethanol acetate (MAM) is a potent alkylating agent that kills mitotically active cells by selectively methylating nucleic acids ${ }^{21}$. MAM has been shown to have neurotoxic effects when administered during the period of active neurogenesis in hamsters, mice, rabbits, rats, beagles, cats and ferrets $^{17}$. Since the compound has a brief half-life in vivo, timing of fetal or perinatal exposure in relation to regional neurogenesis determines the character of CNS malformations ${ }^{17,21}$. In rats, injection of MAM on fetal day 15 eliminates many neurons destined for the outer half of the neocortex but spares important brainstem projections to the neocortex that are formed prior to the date of administration ${ }^{10,11}$. Analysis of DNA in the brain of adult rats treated on day 15 indicate a marked loss of cells in the cerebral hemispheres but not in the brainstem ${ }^{19}$. In addition, neurochemical and immunocytochemical studies of transmitter-specific neuronal populations reveal a marked loss of GABAergic neurons intrin- sic to the hypoplastic cortex but a relative enrichment of noradrenergic, serotonergic and cholinergic terminals derived from the brainstem ${ }^{10-12}$. Varying the fetal age of treatment with MAM in the rat produces a predictable spectrum of changes in the neurochemical and histologic composition of the cortex ${ }^{14}$.

Morphologically, MAM lesions in fetal rats resemble human malformations characterized by lissencephaly, a flattening of the normal gyral pattern of the cortex ${ }^{9,16}$. Gyrencephalic animals such as the ferret provide a better model for these malformations since rat cortex lacks gyri. Previous studies indicate that ferrets exposed to MAM in the last quarter of gestation develop lissencephalic cortices and also exhibit deficits in learning as measured by maze performance ${ }^{6}$. The present study was undertaken to determine whether MAM-induced cortical hypoplasia in the ferret is associated with alterations in neurotransmitter-specific neuronal populations that have been previously described in the rat. Accordingly, we have assayed neurochemical mar- 
kers for GABAergic, cholinergic and catecholaminergic neurons in the neocortex from juvenile ferrets which received MAM on gestational day 33 .

\section{MATERIALS AND METHODS}

\section{Animals}

Time-dated pregnant ferret jills were purchased from Marshall Research Animals (Northrose, New York) and were fed and housed as previously described $^{6}$. Each jill was given a single intraperitoneal injection of either methylazoxymethanol acetate (15 $\mathrm{mg} / \mathrm{kg}$ body weight; Aldrich Chemicals, St. Louis, MO) or an equivalent volume of physiologic saline on day 33 of gestation. Pups were born 9 days later, weaned at 6 weeks of age and sacrificed at 8 weeks of age by decapitation for histologic and neurochemical analysis. Five control and 4 lesioned ferrets from 2 litters each were used in the experiments.

\section{Morphology}

To correlate the anatomical and neurochemical data, brains of animals sacrificed by cervical dislocation were removed from the skull, weighed and divided in half longitudinally. One half was placed in cold $10 \%$ buffered formalin for 3 weeks and then immersed in $30 \%$ sucrose in buffered formalin for 10 days. After photographs were obtained to record the gross appearance of the brain, $40 \mu \mathrm{m}$ coronal sections were obtained on a frozen stage microtome plane and stained with cresyl violet.

\section{Neurochemical analysis}

One-half of the brain was quickly dissected on an ice-cold aluminum block. The hemicortex was isolated by dissecting away all tissue below the cortical white matter. The cortices were then flattened on the aluminum plate with the white matter down and the dorsomedial cingulate and entorhinal cortex were cut away. The remaining cortex was divided into 5 regions as shown in Fig. 1 for neurochemical analysis. Although the gyral landmarks were obscured in the MAM treated cortex, an attempt was made to dissect regions analogous to those of control cortex. The cortical samples were then cut into blocks, which were frozen immediately on dry-ice and stored at $-70^{\circ} \mathrm{C}$ for assay of neurotransmitter synthesizing enzymes or endogenous neurotransmitters.
For neurochemical assays, samples from both agematched control and MAM-lesioned animals were assayed at the same time.

For enzyme assays, the tissue was homogenized in 20 vols. of ice-cold $50 \mathrm{mM}$ Tris- $\mathrm{HCl}, \mathrm{pH} 7.4$ containing $0.2 \%(\mathrm{v} / \mathrm{v})$ Triton $\mathrm{X}-100$. Homogenates were centrifuged at $12,000 \mathrm{~g}$ at $4{ }^{\circ} \mathrm{C}$ for $15 \mathrm{~min}$ and portions of the supernatant were assayed. Activity of choline acetyltransferase was measured by the method of Bull and Oderfeld-Nowak ${ }^{1}$, tyrosine hydroxylase was measured as described by Coyle and Axel$\operatorname{rod}^{2}$ with the exception that D,L-methyl-5,6,7,8-tetrahydropterine- $\mathrm{HCl}$ was used as the co-factor. Glutamate decarboxylase was assayed by a modification of the method of Wilson et al. ${ }^{24}$ with $\mathrm{D}, \mathrm{L}-\left[1-{ }^{14} \mathrm{C}\right] \mathrm{glu}$ tamic acid as substrate with the exception that $\mathrm{pH}$ was lowered to an optimum of 5.5. Protein was measured by the method of Lowry et al. ${ }^{18}$ with bovine serum albumin as standard. To assay the neurotransmitters, the frozen cortex was homogenized in 20 vols. of formic acid-acetone $(15: 85, \mathrm{v} / \mathrm{v})$. Homogenates were centrifuged at $1000 \mathrm{~g}$ for $5 \mathrm{~min}$, and the supernatant was aliquoted into tubes for each assay and dried down in a vacuum centrifuge. Norepinephrine was measured by the method of Coyle and Henry ${ }^{3}$, GABA by the method of Graham and Aprison ${ }^{5}$, and acetylcholine by the method of Goldberg and McCaman ${ }^{4}$. All neurochemical assays were done in triplicate.

\section{RESULTS}

\section{Morphology}

MAM-treatment of ferrets at 33 days gestation resulted in offspring with brain weight reduced by $28 \%$ as compared to age-matched controls (MAM, $5.0 \pm 0.2 \mathrm{~g}$ vs control, $6.9 \pm 0.2 \mathrm{~g} ; P<0.01)$. Although the brainstem and cerebellum appeared relatively normal in the affected offspring, MAM treatment markedly impaired the normal development of the gyral pattern of the cerebral cortex (Fig. 1). The frontal cortical mantle, normally formed by the suprasylvian, middle and sigmoid gyri, was considerably reduced although the most rostral frontal pole was less severely affected. The perisylvian gyral pattern was obliterated and the cortex in the temporal-occipital region was severely hypoplastic and remained as a thin, transparent membrane suppor- 


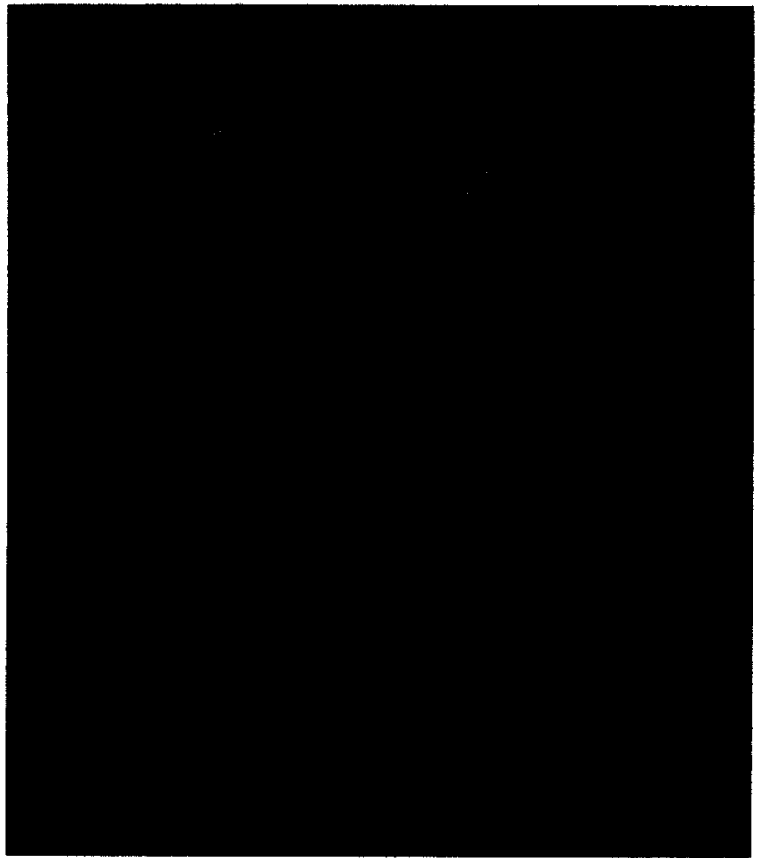

Fig. 1. Gross morphology of the ferret brain. A: the brain of a normal ferret. B: the brain from a ferret treated with MAM at 33 days of gestation. The cortical sub-areas used for neurochemical assays are indicated. Note marked attenuation and ablation of the cortical gyrae in the MAM-treated rat cortex and the sunken temporal cortex resulting from the severe atrophy (asterisk). Abbreviations: F, frontal pole; $P$, prefrontal; L, lateral gyrus; S, suprasylvian gyrus; E, ectosylvian gyrus.

ted in life by the cerebral spinal fluid within the dilated ventricles. Only the presylvian and suprasylvian fissures were readily apparent in most treated animals. Histologic examination of the midlateral cortex of the MAM-treated ferret revealed marked hypoplasia affecting primarily the outermost layers (Fig. 2). Although the molecular layer appeared relatively normal, many solitary neurons and neuronal clumps were ectopically placed in the deep cortex and subcortical regions.

\section{Neurochemistry}

Although there was considerable variation among the subregions of the cortex, the lissencephalic cortex of the MAM-treated ferrets was generally enriched in biochemical markers for cholinergic and catecholaminergic neurons but deficient in markers for the GABAergic neurons (Tables I and II). Notably, no difference in protein content per $\mathrm{mg}$ brain tissue was observed in any regions examined between the treated and control ferrets. In the 5 regions ex- amined, the mean increase in the specific activity of tyrosine hydroxylase, an enzymatic marker for catecholaminergic axons, was $39 \%$; similarly, the concentration of endogenous norepinephrine was elevated on an average of $38 \%$ overall. In contrast, the concentration of endogenous GABA was reduced by an average of $17 \%$ and the specific activity of its synthetic enzyme, glutamate decarboxylase, was reduced by $14 \%$. The specific activity of choline acetyltransferase was increased by $49 \%$ overall whereas endogenous acetylcholine was generally elevated but to a lesser extent.

The presynaptic markers for the catecholaminergic axons in neocortex, tyrosine hydroxylase and endogenous norepinephrine, were both significantly elevated in the prefrontal, ectosylvian and suprasylvian regions, but were less affected in the frontal pole and the lateral gyrus regions. Although the elevations in both markers were comparable in the ectosylvian and suprasylvian regions, it is noteworthy that tyrosine hydroxylase activity was increased to a disproportionately greater extent in the prefrontal region as compared to the concentration of norepinephrine; this disparity may reflect the greater localization of tyrosine hydroxylase in dopaminergic axons within this region.

The reductions in the concentration of endogenous GABA were relatively modest and evenly distributed throughout all regions. These alterations, which reach statistical significance only in the lateral regions, were consistent with the small reductions in the specific activity of glutamate decarboxylase, except in the frontal pole where a $59 \%$ decrease in enzyme activity was noted. Regional alterations in cholinergic markers differed from the pattern observed with the catecholaminergic markers. While the prefrontal section contained the greatest elevations in the specific activity of choline acetyltransferase, the frontal pole was the site of the next highest increase in choline acetyltransferase activity. The increase in choline acetyltransferase activity in the suprasylvian region was virtually identical to those observed with tyrosine hydroxylase and endogenous norepinephrine. Increases in the concentration of endogenous acetylcholine generally occurred in regions in which the activity of its synthetic enzyme was elevated although the alterations in the neurotransmitter were much less marked. 


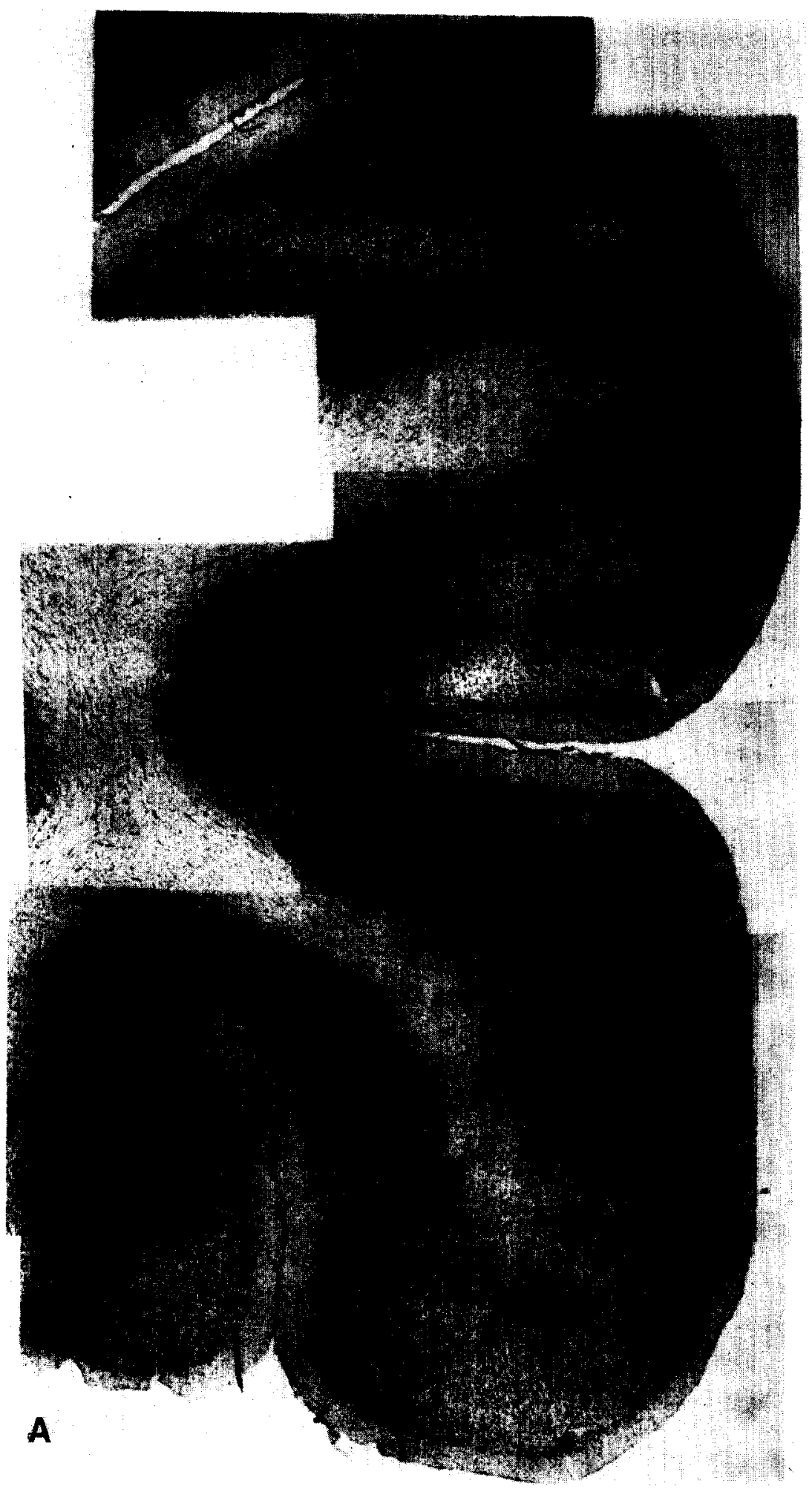

Fig. 2. Histology of the mid-lateral cortex of the ferret. A : normal ferret. B: ferret treated with MAM at 33 days of gestation. Photomontages were obtained from Nissl-stained sections through the mid-lateral cortices from adult control and MAM-lesioned ferrets. Note the absence of gyral organization and the hypoplasia of the external cortical layers in the MAM-treated ferret. 


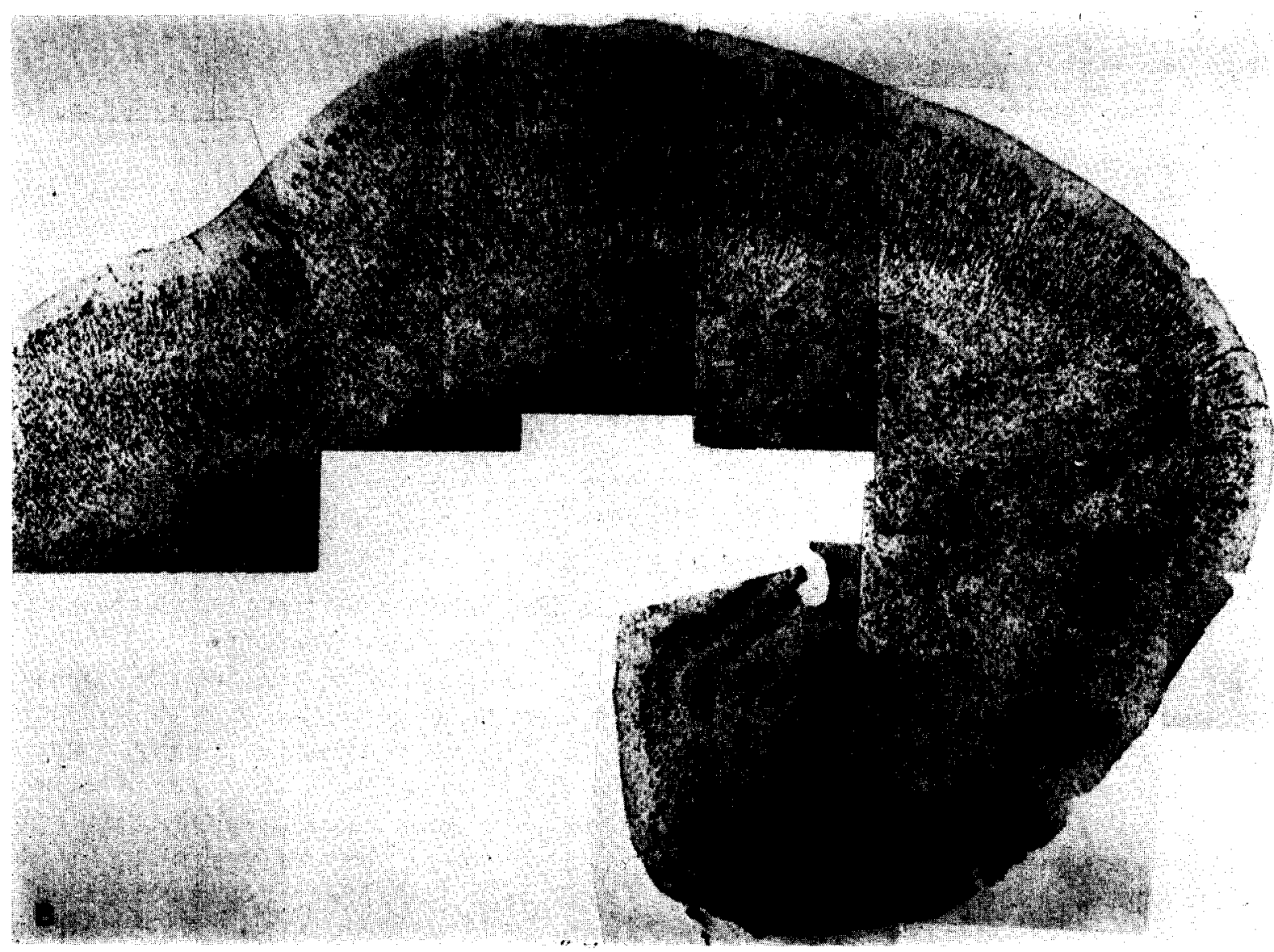

Fig. 2B.

\section{TABLE I}

Neurotransmitter synthesizing enzyme activity in ferret cortex after prenatal treatment with MAM acetate

Enzyme specific activities were determined in cortical regions of the lissencephalic MAM acetate-treated brains and compared with analogous regions shown in Fig. 1. Each value (mean \pm S.E.M.) is the result of duplicate determinations performed in 5 control and 4 treated animals.

\begin{tabular}{|c|c|c|c|c|c|c|c|c|c|}
\hline \multirow[t]{2}{*}{ Area } & \multicolumn{3}{|c|}{$\begin{array}{l}\text { Tyrosine hydroxylase } \\
\left(\mathrm{pmol} \cdot \mathrm{h}^{-1} \cdot \mathrm{mg}_{\text {prot }}{ }^{-1}\right)\end{array}$} & \multicolumn{3}{|c|}{$\begin{array}{l}\text { Choline acetyltransferase } \\
\left(\text { nmol } \cdot h^{-1} \cdot m \text { prot }^{-1}\right)\end{array}$} & \multicolumn{3}{|c|}{$\begin{array}{l}\text { Glutamate decarboxylase } \\
\left(\text { nmol }^{\prime} h^{-1} \cdot \mathrm{mg}_{\text {prot }}{ }^{-1}\right)\end{array}$} \\
\hline & Control & Treated & $\Delta \%$ & Control & Treated & $\Delta \%$ & Control & Treated & $\Lambda \%$ \\
\hline Frontal pole & $28 \pm 2$ & \pm 1 & +11 & $16.6 \pm 2$ & $28.1 \pm 2^{*}$ & +61 & $7.1 \pm 0.8$ & $2.9 \pm 0.8^{* *}$ & -59 \\
\hline Prefrontal & $53 \pm 4$ & $91 \pm 7 * *$ & +72 & $19.4 \pm 2$ & $42.3 \pm 6^{* *}$ & +118 & $5.9 \pm 0.3$ & $5.9 \pm 0.3$ & 0 \\
\hline Lateral gyrus & $26 \pm 3$ & $31.6 \pm 2$ & +16 & $16.5 \pm 2$ & $16.5 \pm 3$ & 0 & $6.5 \pm 0.4$ & $6.2 \pm 0.8$ & -5 \\
\hline Suprasylvian gyrus & $36 \pm 2$ & $50 \pm 5^{*}$ & +39 & $17.3 \pm 1$ & $24.1 \pm 1^{* *}$ & +39 & $5.9 \pm 0.4$ & $5.7 \pm 0.3$ & -3 \\
\hline Ectosylvian gyrus & $27 \pm 2$ & $\pm 3^{* *}$ & +59 & $33.5 \pm 3$ & $43.0 \pm 6$ & +28 & $7.5 \pm 0.3$ & $7.2 \pm 0.7$ & -4 \\
\hline
\end{tabular}

$* P<0.05 ; * * P<0.01 ;$ control vs treated (Student's $t$-test). 
TABLE II

Endogenous neurotransmitter levels in ferret cortex after prenatal treatment with MAM acetate

Endogenous neurotransmitter concentrations were determined in regions of untreated cortex (Fig. 1) and analogous regions of lissencephalic MAM acetate-treated cortex. Each value (mean \pm S.E.M.) is the result of duplicate determinations performed in 5 control and 4 treated animals. ND, not determined.

\begin{tabular}{|c|c|c|c|c|c|c|c|c|c|}
\hline \multirow[t]{2}{*}{ Area } & \multicolumn{3}{|c|}{ Norepinephrine (pg/mg tissue) } & \multicolumn{3}{|c|}{$G A B A$ (nmol/mg tissue) } & \multicolumn{3}{|c|}{ Acetylcholine (pmol/mg tissue) } \\
\hline & Control & Treated & $4 \%$ & Control & Treated & $\Delta \%$ & Control & Treated & $\Delta \theta_{0}$ \\
\hline Frontal pole & $150 \pm 25$ & $170 \pm 26$ & +11 & $1.29 \pm 0.04$ & $1.19 \pm 0.02$ & -8 & $12.6 \pm 0.6$ & $13.8 \pm 0.08$ & +10 \\
\hline Prefrontal & $150 \pm 20$ & $210 \pm 4^{*}$ & +40 & $1.26 \pm 0.03$ & $1.04 \pm 0.09$ & -17 & $10.4 \pm 0.4$ & $14.9 \pm 1.5^{*}$ & +43 \\
\hline Lateral gyrus & $190 \pm 17$ & $220 \pm 30$ & +16 & $1.24 \pm 0.05$ & $1.01 \pm 0.07$ & -19 & $12.0 \pm 0.6$ & $13.6 \pm 0.05$ & +13 \\
\hline Suprasylvian gyrus & $152 \pm 22$ & $219 \pm 9^{*}$ & +44 & $1.25 \pm 0.06$ & $1.00 \pm 0.06^{*}$ & -20 & $10.8 \pm 0.03$ & $9.5 \pm 1.4$ & -12 \\
\hline Ectosylvian gyrus & $140 \pm 13$ & $250 \pm 23^{* *}$ & +80 & $1.32 \pm 0.04$ & $1.06 \pm 0.01 * *$ & $*-21$ & ND & & \\
\hline
\end{tabular}

${ }^{*} P<0.05 ;{ }^{* *} P<0.01$; control vs treated (Student's $t$-test).

\section{DISCUSSION}

The results of the present experiments with fetal MAM lesions of ferret are generally congruent with our previous findings in the rat which have shown significant imbalances among cortical neurotransmitter systems ${ }^{10-12,14,15,20,23}$. Although the most characteristic neuroanatomic feature of the lesioned cortex is hypoplasia, the neurochemical analyses reveal supernormal concentrations of markers for cortical catecholaminergic and cholinergic terminals. The presynaptic markers for GABAergic neurons were generally unchanged or mildly reduced in concentration but markedly depressed when considered in terms of their total amounts per cortex.

Previous studies in the rat indicate that the shortacting alkylating agent MAM selectively ablates neurons in the CNS which are dividing at the time of administration $7,14,19,21$. Because the neurons making up the neocortex divide relatively late in gestation $^{8}$, rather selective deletions of neocortical laminae can be produced by administration of MAM when most brainstem systems have ceased dividing and have begun differentiation. Similarly, administration of MAM at the beginning of the last week of gestation for the ferret results in a marked cortical hypoplasia whereas the brainstem and cerebellum are relatively spared ${ }^{6}$. An important distinction between the gyrencephalic ferret and the lissencephalic rat concerns the disruption of normal gyral formation in the ferret after the late fetal MAM lesion. Thus, the effects of the lesion in ferret is not simply restricted to cortical thickness but macroscopic aspects of neocortical organization.

The neocortical GABAergic neurons, aspinous stellate cells, are evenly distributed throughout all cortical layers ${ }^{22}$. Congruent with this distribution, neurochemical analysis in the cortex of the fetal MAM-lesioned rat indicated no alteration in the concentration of presynaptic markers for the GABAergic neurons but a reduction directly proportional to the degree of cortical hypoplasia. In contrast, the presynaptic markers for the noradrenergic terminals innervating the cortex were increased in concentration in the MAM-lesioned rat cortex in inverse proportion to the degree of hypoplasia; this reflected the quantitatively normal development of the noradrenergic terminal arbor which was compressed in the reduced cortical volume ${ }^{10,11}$. A similar enrichment in the presynaptic markers of the cholinergic terminals was noted in the MAM-induced hypoplasia of the rat neocortex although the total concentration of the cholinergic markers were significantly reduced consistent with the evidence for a population of cortical cholinergic intrinsic neurons that would be affected by the MAM lesion.

Although the regional morphologic-neurochemical analysis in the MAM-lesioned ferret cortex was generally consistent with the findings in the rat, certain dissimilarities are also apparent. It was not possible in the ferret to determine the total amount of markers per cortical mantle; nevertheless, a simple inverse relationship between the concentration of noradrenergic terminal markers and the degree of 
hypoplasia of subregions of the cortex did not appear to hold. While regions of the ferret cortex which were markedly affected morphologically (prefrontal, supersylvian and ectosylvian regions) exhibited the largest increase in catecholaminergic markers, other clearly affected regions such as the lateral gyrus exhibited only modest elevations that were not consistent with the degree of hypoplasia. Similarly, the elevations in the presynaptic cholinergic markers, while greatest in the severely affected area, the prefrontal region, also exhibited a substantial increase in the frontal pole, which was less severely affected. Curiously, the frontal pole also exhibited a significant reduction in the specific activity of glutamate decarboxylase, although the concentration of

\section{REFERENCES}

1 Bull, G. and Oderfeld-Nowak, B., Standardization of a radiochemical assay of choline acetyltransferase and a study of the activation of the enzyme in rabbit brain, $J$. Neurochem., 18 (1971) 935-947.

2 Coyle, J. T. and Axelrod, J., Tyrosine hydroxylase in rat brain: developmental characteristics, J. Neurochem., 19 (1972) 1117-1123.

3 Coyle, J. T. and Henry, D., Catecholamines in fetal and newborn rat brain, J. Neurochem., 21 (1973) 61-67.

4 Goldberg, A. M. and McCaman, R. E., The determination of picomole amounts of acetylcholine in mammalian brain, J. Neurochem., 20 (1973) 1-8.

5 Graham, L. T. and Aprison, M. H., Fluorometric determination of aspartate, glutamate, $\gamma$-aminobutyric acid in nerve tissue using enzymatic methods, Analyt. Biochem., 15 (1966) 487-497.

6 Haddad, R., Rabe, A. and Dumas, R., Neuroteratogenicity of methylazoxymethanol acetate: behavioral deficits of ferrets with transplacentally induced lissencephaly, Neurotoxicology, 1 (1979) 171-189.

7 Haddad, R. K., Rabe, A., Laquer, G. L., Spatz, M. and Valsamis, M., Intellectual deficit associated with transplacentally induced microcephaly in the rat, Science, 163 (1969) 88-90.

8 Hicks, S. J. and D'Amato, C. J., Cell migrations to isocortex in the rat Anat. Rec., 160 (1968) 619-634.

9 Jellinger, K. and Rett, A., Agyria-pachygyria (Lissencephaly syndrome), Neuropadiatrie, 7 (1976) 66-91.

10 Johnston, M. V. and Coyle, J. T., Histological and neurochemical effects of fetal treatment with methylazoxymethanol on rat neocortex in adulthood, Brain Res., 170 (1979) 135-155.

11 Johnston, M. V. and Coyle, J. T., Ontogeny of neurochemical markers for noradrenergic, GABAergic, and cholinergic neurons in neocortex lesioned with methylazoxymethanol acetate, J. Neurochem., 34 (1980) 14291441.

12 Johnston, M. V., Grzanna, R. and Coyle, J. T., Abnormally dense noradrenergic innervation of rat neocortex follows fetal treatment with methylazoxymethanol, Science, 203 (1979) 369-371.

13 Johnston, M. V., McKinney, M. and Coyle, J. R., Evidence for a cholinergic projection to neocortex from neu-
GABA itself was minimally depressed. The factors contributing to the significant differences in the synaptic neurochemical sequelae of the MAM-induced hypoplasia of the ferret neocortex as compared to that of the rat remain unclear but may reflect the much greater extent of encephalization in the ferret.

\section{ACKNOWLEDGEMENTS}

This research was supported by USPHS Grants MH 26654, MH 00125 and DA 00266, and grants from the March of Dimes and the McKnight Foundation.

We thank Carol Kenyon for excellent secretarial assistance.

rons in basal forebrain, Proc. nat. Acad. Sci. (U.S.A.), 76 (1979) 5392-5396.

14 Johnston, M. V., Carman, A. B. and Coyle, J. T., Effects of fetal treatment with methylazoxymethanol acetate at various gestational dates on the neurochemistry of the adult neocortex of the rat, $J$. Neurochem., 36 (1981) 124-128.

15 Johnston, M. V., Young, A. C. and Coyle, J. T., Laminar distribution of cholinergic markers in neocortex: effects of lesions, J. Neurosci. Res., 6 (1981) 597-607.

16 Larroche, J. C., Cytoarchitectonic abnormalities (abnormalities of cell migration). In P. J. Vinken and G. W. Bruyn (Eds.), Handbook of Clinical Neurology, Vol. 30, 1977, pp. 477-506.

17 Laquer, G. L., Oncogenecity of cycads and its implications. In H. F. Kraybill and M. A. MehIman (Eds.), Advances in Modern Toxicology: Environmental Cancer, Vol. 3, Wiley, New York, 1977, pp. 231-261.

18 Lowry, O. H., Rosebrough, N. J., Farr, A. L. and Randall, R. J., Protein measurement with Folin phenol reagent, J. biol. Chem., 193 (1951) 265-275.

19 Matsumoto, H., Spatz, M. and Laquer, G. L., Quantitative changes with age in the DNA content of methylazoxymethanol-induced microencephalic rat brain, $J$. Neurochem., 19 (1972) 297-306.

20 Matsutani, T., Nagayoshi, M., Tamaru, M. and Tsukada, Y., Elevated monoamine levels in the cerebral hemispheres of microencephalic rats treated prenatally with methylazoxymethanol or cytosine arabinoside, $J$. Neurochem., 34 (1980) 950-956.

21 Nagata, Y. and Matsumoto, H., Studies on methylazoxymethanol: methylation of nucleic acids in fetal rat brain, Proc. Soc. exp. Biol. (N.Y.), 132 (1969) 383-385.

22 Ribak, C. E., Aspinous and sparsely-spinous stellate neurons in the visual cortex of rats contain glutamic acid decarboxylase, Neurocytology, 7 (1978) 461-478.

23 Slevin, J. T., Johnston, M. V., Biziere, K. and Coyle, J. T., Methylazoxymethanol acetate ablation of mouse cerebellar granule cells: effect on synaptic neurochemistry, Develop. Neurosci., (1981) in press.

24 Wilson, S. H., Schrier, B. K., Farber, J. L., Thompson, E. J., Rosenberg, R. M., Blume, A. J. and Nirenberg, M. W., Markers for gene expression in cultured cells from the nervous system, J. biol. Chem., 247 (1972) 3159-3169. 